\title{
Journal of Pregnancy and Child Health
}

\section{Women Refusing Standard Obstetric Care: Maternal Fetal Conflict or Doctor- patient Conflict?}

\author{
Martine Hollander $^{1^{*}}$, Jeroen van Dillen ${ }^{1}$, Toine Lagro-Janssen ${ }^{2}$, Evert van Leeuwen ${ }^{3}$, Wilma Duijst ${ }^{4}$ and Frank Vandenbussche ${ }^{1}$ \\ ${ }^{1}$ Department of Obstetrics and Gynaecology, Radboud University Medical Center, Nijmegen, the Netherlands \\ ${ }^{2}$ Department primary and community care, unit gender and women's health, Radboud University Medical Center, Nijmegen, the Netherlands \\ ${ }^{3}$ Department of IQ Healthcare, Radboud University Medical Center, Nijmegen, the Netherlands \\ ${ }^{4}$ Department of Forensic Medicine, GGD IJsselland, Zwolle, the Netherlands and district court, Arnhem, the Netherlands \\ *Corresponding author: Martine Hollander, Radboud University Nijmegen Medical Centre, Nijmegen, Netherlands, Tel: 31612858004; E-mail: \\ martine_hollander@hotmail.com
}

Received date: April 26, 2016; Accepted date: May 17, 2016; Published date: May 24, 2016

Copyright: ( $) 2016$ Hollander M, et al. This is an open-access article distributed under the terms of the Creative Commons Attribution License, which permits unrestricted use, distribution, and reproduction in any medium, provided the original author and source are credited.

\begin{abstract}
Objectives: Some women choose to give birth outside medical protocol or ignore medical advice. This could be perceived as a maternal-fetal conflict. Many professionals are unsure about the legal possibilities and ethical intricacies in these circumstances, and the position of the fetus. This paper attempts to elucidate and provide a framework for these issues.
\end{abstract}

Methods: We did a literature search on Pubmed in October 2014, using the terms "legal/law", "pregnant/ pregnancy", "legal measures", "court-ordered caesarean/cesarean", "birth", "childbirth" and "homebirth". We also reviewed some professional organisations' guidelines.

Results: Much has been written about legal restrictions and measures against women who go against medical advice, for instance court-ordered cesarean sections or forced hospital admissions. Medical professionals fear litigation in case of a bad outcome when the mother's wishes are respected. However, medical assessment of risk and benefit is sometimes inaccurate. Maternal competence is a central issue.

The pregnant patient has the right to autonomy, bodily integrity, freedom and self-determination and the fetus has the right to have its life protected. However, it is unethical to invade one person's physical integrity for the benefit of another, especially if the other is not born yet. Religious rights are generally respected, but this is not unlimited.

International guidelines on this subject generally state that a competent pregnant patient has the same rights as any other person and that her autonomy should prevail.

Conclusions: In contrast to the general perception the conflict described is not between mother and child but between doctor and patient. Communication can be the key to solving this problem. In cases of continued disagreement, the mother's autonomy should prevail.

Significance: In this era of social media and access to information for all, patients are better informed than ever before. At the same time, shared decision making is rapidly becoming the norm. Obstetrical care providers are increasingly faced with pregnant patients who refuse some or all proposed interventions. These decisions may appear to be at odds with what medical professionals deem best for the fetus. We have attempted to provide a legal and ethical framework for looking at this dilemma.

Keywords: Conflict, Legal, Ethical, Mother, Fetus

\section{Introduction}

Evidence based guidelines can help healthcare practitioners to provide better and more cost effective care for the average patient with a specific medical problem [1]. During the past 15 years, the Cochrane Collaboration, NICE, and many other national and international organizations have provided obstetric medicine with a wealth of new evidence based guidelines. However, more protocolized care also means less room for personalized medicine. Not all patients have the same perception of risk as medical staff [2] and some attach more importance to avoiding a certain intervention (for instance caesarean section) than to incurring a small increase in risk of perinatal morbidity or mortality. One of the consequences of the plethora of protocols in obstetric medicine could be a real or perceived increase in the number of patients who wish to go outside the standard of care. No quantitative surveys on this subject have been done to date, so exact numbers are not available. Much is still unclear among professionals about the rights of a pregnant woman, those of her fetus, and the legal position of a healthcare provider who is willing to assist a woman who wants to give birth outside the standard protocol. The debate is as yet unresolved whether the law can or indeed should intervene in situations where the woman's decision seems to put the fetus's life at 
risk. This is often described by obstetricians as a maternal-fetal conflict. In this paper we will provide some insight into the legal and ethical context surrounding women's rights in childbirth and the issues mentioned above. We will also review some professional organizations' statements on these issues. Legal measures against pregnant women in literature.

For the purposes of this paper, we were interested in the legal position of women who desire to give birth outside the current medical protocols. This constitutes giving birth at home against medical advice, or giving birth unattended by any medical professional (free birth or unattended childbirth). We did a literature search on Pubmed in October 2014, using the terms "legal/law", "pregnant/pregnancy", "legal measures", "court-ordered caesarean/caesarean", "birth", "childbirth" and "homebirth". We found many publications detailing how women's choices in pregnancy and childbirth have been restricted and punished by both criminal and civil law. A full review of this issue was written by Cherry [3] and provided many of the examples used below. Much of this literature originates in the United States and concerns civil law. In the 1980's and 90's a substantial number of papers were written about court-ordered caesareans (COC). The first reported case occurred in 1981, and concerned a patient with complete placenta previa [4]. She refused caesarean section on religious grounds and a court order was obtained to enforce the procedure, citing a state interest in the welfare of the term fetus. However, before the section could be performed she went into labour and delivered vaginally without major problems. Another well-known case is the one of Laura Pemberton, a Florida woman who attempted a home birth after a previous caesarean section. Her doctors judged that she needed a repeat caesarean and obtained a court order from a judge to have her forcibly removed from her home. She underwent a caesarean section under protest and subsequently had three more vaginal deliveries [5]. Cherry reports a case of a woman being arrested for fear of her giving birth unattended by a health care professional [5]. In 1987, Kolder et al. [6] published the first review of known COC cases. They also included one case of forced blood transfusion to a pregnant Jehovah's Witness. Notably, a large proportion of the patients who had legal action taken against them for non-compliance with doctors' recommendations was poor, black, unmarried and on welfare.

It is notoriously difficult for medical professionals to accurately predict fetal outcome. Annas, in 1982, described two cases of COC in the United States. The first case involved another woman with placenta previa who was judged by her doctors as having $99 \%$ chance of fetal death in case of a vaginal delivery. A COC was ordered, but in the mean time she delivered vaginally and had a healthy child. In the second case the attending obstetrician diagnosed fetal distress. The COC was performed nine hours later and the child was born in good condition [7]. Recently, a Florida court compelled a pregnant woman to undergo a cesarean section against her wishes after premature rupture of membranes at 25 weeks. She had wanted to go home and get a second opinion but her physician sought and obtained a court order. The COC was performed three days later and resulted in a stillbirth [8]. In contrast, Elkins reported two cases in 1989, one of which was an unwanted pregnancy where a COC was sought for fetal distress. It was denied and an intra-partum fetal death occurred. In the other case, the pregnant woman was diagnosed with severe depression. A COC was performed for fetal distress and the baby was born alive but acidotic. Both of these women were in the hospital for the first time and had not had any chance of discussing their wishes prior to the described emergency [9].
Many other interventions and measures against pregnant women have been reported in criminal law literature. In the United States, some states can give the court jurisdiction over unborn children when the mother habitually uses drugs or alcohol $[5,10]$. This means that pregnant women can be taken into custody and sentenced to prison for child endangerment when they use illicit substances. This has led to 12 states requiring physicians to report drug use in pregnancy to child welfare agencies [3]. We have been unable to find any reference to action being taken against a pregnant woman for smoking cigarettes or being overweight, even though these are also well established risk factors for a number of unfavourable outcomes. However, documented cases include women being arrested for exposing their unborn child to dangerous fumes, for not following doctors' orders to take bed rest, and for taking a long time to get to the hospital while in labour or while bleeding [1,5]. Cherry [3] describes a case where a judge sentenced a pregnant woman to prison for credit card fraud to prevent her from having the opportunity to obtain the abortion she announced she wanted. By 1999, more than 200 US women were on record for having been arrested for endangering fetal health.

Legal measures have also been taken after the fact: several mothers have been charged with murder after refusing a caesarean section deemed necessary to save the life of their child [11-14]. Bowes, in 1981, cited jurisprudence where "the fetus may be the victim of homicide if born alive but dies as a result of prenatal injury [15]. And even if the child survives, it can later sue it's mother in civic court for actions taken by her that may have adversely affected the child's development prior to birth [11]. This paves the way for any number of legal issues surrounding choices pregnant women, or indeed parents, make regarding their (unborn) children. Although less has been published on this subject in recent years, court-ordered caesarean sections are by no means a thing of the past. Margolin described a case in 2014 in Israel where medical staff was given carte blanche by the court to perform any examination or intervention deemed necessary in order to prevent damages to the fetus and the patient herself [16]. In a survey performed among the heads of maternal-fetal medicine fellowships in the United States in 1987, many thought that pregnant women who endangered their fetus's life should be detained and that forced treatment under those circumstances was acceptable [6]. Adams in 2003 and Samuels in 2007 did similar surveys and found that, although the willingness to go against patient's wishes by taking legal action had significantly decreased, still every interviewee could envision conditions under which they would ultimately take such a step [13,17].

In the matter of legal involvement with women's decisions in pregnancy, competence is a central issue. When unconscious, a patient is incompetent. In that case, consent can be assumed for interventions deemed necessary to prevent death or serious harm to the patient. Competence can also be in question in cases of severe mental illness or a state of drug-induced decreased mental capacity $[18,19]$ although this does not necessarily imply that a patient with any psychiatric problem is automatically legally incompetent. In addition, the vast majority of pregnant women, regardless of whether or not they agree with the treatment their physician suggests, are legally competent. However, some physicians find it difficult to imagine how a person of sound mind could possibly disagree with the proposed plan of treatment. Cahill wrote about this in 1999: "There seems to be a blanket assumption of maternal incompetence: women who refuse recommended treatment cannot be of sound mind [20]. But, as Cherry [3] states: "the making of poor choices does not generally constitute mental disease or defect". 
In this era of malpractice lawsuits, physicians are, understandably afraid when patients make choices that, in the opinion of the doctor, may increase the chance of harm to the fetus. However, we have found no reported cases in the literature where a health care professional was found guilty of negligence by a court for respecting a competent patient's wishes [21]. Therefore these fears appear to be unfounded. On the other hand, overruling the patient may leave the treating physician open to allegations of assault and battery [22]. Legal concerns have led doctors to be guided by fear and to practice defensive medicine [23]. Many daily obstetrical decisions are made based on risks, without knowing exactly how high those risks are. Medicine itself seems to be moving more and more towards a risk based, preventative approach, where pathology is defined by a standard deviation of the norm. A recent survey done among young (mostly female) obstetricians in Canada showed that they, compared to their older, predominantly male predecessors, were more likely to favor technology during birth in order to maintain control, and were less appreciative of the role of women in their own birth [24].

\section{Ethical Considerations: Do the Rights of the Mother Conflict with those of the Child?}

Informed consent and shared decision making are important principles of current medical practice. Health care professionals outline the different treatment options with the accompanying risks and benefits, and the patient decides. Although the patient may make another decision than the doctor would make, this has not led to doctors forcing patients to undergo surgery or a medical therapy that the patient does not wish, even if it leads to the untimely demise of the patient. Obstetrics is different and unique in the sense that many health care professionals feel that there are two patients involved: mother and fetus, and doctors feel they are equally responsible for both. If the mother makes a decision that the professional feels may put her child at risk, it may be emotionally difficult to accept this. In the greater part of the twentieth century, the aim of prenatal care in the developed world was reducing maternal mortality. In recent decades, with the arrival of ultrasound and fetal monitoring, the fetus has become much more visible during pregnancy and has now become the main focus of prenatal care. Pregnant patients are offered genetic counseling, fetal aneuploidy screening and ultrasounds for structural defects. It seems natural that, with increasing visibility of the fetus, more importance is being attached to its rights and wellbeing. In weighing the wishes of the mother against fetal interests, numbers needed to treat and numbers needed to harm play a role, as well as the valuation of the different outcomes. It is a well-known fact that medical professionals frequently disagree amongst each other in the estimation of risks and benefits, and also in their advice [6]. If a decision that a pregnant woman makes with regard to her pregnancy increases the chances of fetal harm by a certain margin that is not the same as saying that the fetus will certainly come to harm.

Autonomy, bodily integrity, freedom and self-determination are important principles in modern society. Pregnant women should be no exception. However, freedom and self-determination are not absolute. They can be curtailed if necessary to prevent harm to others, for instance in the case of mandatory isolation during an outbreak of an infectious disease [25]. With growing medical knowledge of the fetal condition in utero there are more arguments being made for fetal rights [26]. The question then becomes: is the fetus an "other" in the eyes of the law [22]? This leads directly to the debate on personhood. What constitutes a person? The law in many countries states that one has to be born to be a person. However, the case for personhood for the fetus is, counter-intuitively, largely grounded in abortion law. If abortion is illegal after viability is attained, does this not automatically mean that the fetus at that point gains certain rights to have its life protected? And if a woman does not choose abortion, has she de facto accepted that the fetus is a person and even subordinated her personal rights to the fetus's right to life? This is described by Draper [27] as a slippery slope: we do defend the rights of the fetus in the respect that we can't end its life any time the mother wants, but as long as it is not born, the mother's wish prevails.

Next we should look at forced interventions on pregnant women for the benefit of their fetus. In order to protect one from harm, we would have to do harm to the other. The question is: is it ethical to inflict harm on one person against their will to prevent harm to another? Some have attempted to answer this question by comparing the maternal fetal "conflict" to that of one person being forced to donate an organ for the benefit of another (the US legal case of McFall vs Shimp). This reasoning has never been approved by a court, not even in the case of a deceased person who during life had chosen not to become an organ donor after death. Do we then award pregnant women fewer rights than deceased people or fetuses more rights than people who have already been born? Of course, this comparison is unfair to the extent that, in most cases, the harm to the mother would be temporary and would not leave any lasting physical scars, with the exception of a forced caesarean section. However, if we would, under certain circumstances, be willing to take away the mother's autonomy for the benefit of her fetus, where would that lead us? Forced bed rest for threatening preterm labour? Mandatory cessation of smoking in pregnancy, punishable by fines or incarceration?

Some women have claimed religion as a reason for refusing to follow medical advice [4]. Although freedom of religion is an important right in modern society, religious rights have also been the focus of much social debate. For instance, male circumcision is widely accepted in many western countries as either a cultural or religious requirement, whereas female genital mutilation, for those same reasons, has generally been outlawed, even in countries where it is widely practiced. Therefore, there are limits to freedom of religion, where it concerns decisions parents make for their children. Whether or not a child is actually born can be a deciding factor. For instance, a Jehovah's Witness can refuse a blood transfusion for herself, even when she is pregnant. However, once her child is born and needs a blood transfusion to survive, a court may relieve the parents of their parental rights in order to be able to override their refusal. If, for example, a 36 weeks pregnant rhesus negative woman with decreased fetal movements comes to the hospital and ultrasound reveals severe fetal anaemia, she may refuse an intrauterine transfusion, based on religious reasons. But how would we value her decision if, after consultation with her religious leaders, she also refuses induction of labour in order for a paediatrician to perform a neonatal transfusion?

\section{Guidelines by Professional Organizations Regarding the Issue of Maternal-fetal Conflict}

Many professional organizations have created guidelines on how to handle situations of perceived maternal-fetal conflict. The American Congress of Obstetricians and Gynaecologists states: "Pregnant women's autonomous decisions should be respected. Concerns about the impact of maternal decisions on fetal well-being should be discussed in the context of medical evidence and understood within the context of each woman's broad social network, cultural beliefs, and 
values. In the absence of extraordinary circumstances, circumstances that, in fact, the Committee on Ethics cannot currently imagine, judicial authority should not be used to implement treatment regimens aimed at protecting the fetus, for such actions violate the pregnant woman's autonomy" [28]. The Australian Medical Association says: "A pregnant woman has the same rights to privacy, to bodily integrity, and to make her own informed, autonomous health care decisions as any competent individual, consistent with the legal framework of that jurisdiction. A pregnant woman's capacity to make an informed decision should not be confused with whether or not the doctor (medical practitioner) considers her decision to be reasonable, sensible or advisable. A doctor may not treat a competent pregnant woman who has refused consent to treatment. Recourse to the law to impose medical advice or treatment on a competent pregnant woman is inappropriate [29]. In addition, the American Academy of Pediatrics counsels that "court intervention should be sought only in rare cases and should be seen as a last resort to be undertaken with great caution [30]. The Royal College of Obstetricians and Gynaecologists in the United Kingdom finds that: "It is inappropriate and unlikely to be helpful or necessary to invoke judicial intervention to overrule an informed and competent woman's refusal of a proposed medical treatment, even though her refusal might place her life and that of her fetus at risk" [31]. Recently, the World Health Organization released a statement on "The prevention and elimination of disrespect and abuse during facility-based childbirth [32].

\section{Conclusion}

Medical professionals working in obstetrics often feel as if there are not one but two patients to consider: the pregnant woman and her unborn child. In recent years, the way in which medicine is practiced has changed, due to increased evidence and protocols. Where the medical professional trusts in the protocol, some patients may feel more comfortable putting their faith in their own body's ability to give birth without (a certain amount of) medical intervention. As shown in the examples mentioned in this paper, professionals frequently disagree on the preferred course of action and are sometimes incorrect in their estimation of fetal danger. In some cases this may lead to a situation where doctor and patient disagree on the treatment plan. If the patient opts for a course that could lead to a perceived increase in risk for the fetus, the doctor may feel that there is a conflict between mother and fetus, where in reality it is the doctor and the patient who are at odds. The best solution to this problem is not to be found in legal action taken against the mother, but in communication between doctor and patient. Counseling patients with respect for their individual circumstances, background, opinions and convictions, and being open and honest about risks, benefits, and uncertainty, will in most cases be sufficient to resolve the problem. In some select cases, an agreement cannot be reached. In those cases, it may be advisable to discuss the patient's wishes in a multidisciplinary setting. Panelists could be obstetricians, midwives, nurses, legal and ethical experts and social workers, and even the patient herself. The aim of such a discussion is to attempt to reach a compromise with which both patient and medical staff feel comfortable. If a compromise cannot be reached, the autonomy of the patient, according to professional guidelines, should prevail.

\section{References}

1. Talaulikar V, Nagarsekar U (2012) Evidence-Based Medicine: An Obstetrician and Gynaecologist's Perspective. J Obstet Gynaecol India 62: 146-153.

2. Jackson M, Dahlen H, Schmied V (2012) Birthing outside the system: perceptions of risk amongst Australian women who have freebirths and high risk homebirths. Midwifery 28: 561-567.

3. Cherry A (2007) The Detention, Confinement, and Incarceration of Pregnant Women for the Benefit of Fetal Health. Cleveland-Marshall College of Law.

4. Finamore EP (1983) Jefferson v. Griffin Spalding County Hospital Authority: court-ordered surgery to protect the life of an unborn child. Am J Law Med 9: 83-101.

5. Paltrow LM (2013) Roe v Wade and the new Jane Crow: reproductive rights in the age of mass incarceration. Am J Public Health 103: 17-21.

6. Kolder VE, Gallagher J, Parsons MT (1987) Court-ordered obstetrical interventions. N Engl J Med 316: 1192-1196.

7. Annas GJ (1982) Forced cesareans: the most unkindest cut of all Hastings Cent Rep 12: 16-1745.

8. Charles S (2011) Obstetricians and violence against women. Am J Bioeth 11: 51-56.

9. Elkins TE, Andersen HF, Barclay M, Mason T, Bowdler N, et al. (1989) Court-ordered cesarean section: an analysis of ethical concerns in compelling cases. Am J Obstet Gynecol 161: 150-154.

10. Ikemoto LC (1998) Forced cesareans. Curr Opin Obstet Gynecol 10: 465-468.

11. Johnsen D (1987) A new threat to pregnant women's autonomy. Hastings Cent Rep 17: 33-40.

12. Symon A, Winter C, Dip P, Donnan P, Kirkham M (2010) Examining Autonomy's Boundaries: A Follow-up Review of Perinatal Mortality Casus in UK Independent Midwifery. Birth 37:4.

13. Samuels TA, Minkoff H, Feldman J, Awonuga A, Wilson T (2007) Obstetricians health attorneys, and court-ordered cesarean sections. Women's Health Issues 17: 107-114.

14. Marwick C (2004) Mother accused of murder after refusing caesarean section. BMJ 328: 663.

15. Bowes WA, Selgestad B (1981) Fetal versus maternal rights: medical and legal perspectives. Obstet Gynecol 58: 209-214.

16. Margolin J, Mester R (2014) Medical treatment without patient's consent and against her will. Harefuah 153: 139-141.

17. Adams SF, Mahowald MB, Gallagher J (2003) Refusal of treatment during pregnancy. Clin Perinatol 30: 127-140.

18. Hondius A, Stikker T, Wennink J, Honig A (2011) The law for special admission for psychiatric illness applied to an addict in early pregnancy. Ned Tijdschr Geneeskd 155: 3818.

19. Schneider AJ, Raats ME, Blondeau MJ, Steegers EA (2004) Pregnant, addicted prostitutes: compulsory admission is sometimes necessary in the interests of the child. Ned Tijdschr Geneeskd 148: 1949-1952.

20. Cahill H (1999) An Orwellian scenario: court ordered caesarean section and women's autonomy. Nurs Ethics 6: 494-505.

21. Nelson LJ (1992) Legal dimensions of maternal-fetal conflict. Clin Obstet Gynecol 35: 738-748.

22. Rossiter G (1995) Contemporary transatlantic developments concerning compelled medical treatment of pregnant women. Aust NZ J Obstet Gynaecol 35: 132-8.

23. Bishop TF, Federman AD, Keyhani S (2010) Physicians' views on defensive medicine: a national survey. Arch Intern Med 170: 1081-1083.

24. Klein MC, Liston R, Fraser WD, Baradaran N, Hearps SJ, et al. (2011) Attitudes of the new generation of Canadian obstetricians: how do they differ from their predecessors? Birth 38: 129-139.

25. CDC (2015).

26. Thampapillai D (2005) Court-ordered obstetrical intervention and the rights of a pregnant woman. J Law Med 12: 455-461. 
Citation: Hollander M, Dillen JV, Janssen TL, Leeuwen EV, Duijst W, et al. (2016) Women Refusing Standard Obstetric Care: Maternal Fetal Conflict or Doctor-patient Conflict?. J Preg Child Health 3: 251. doi:10.4172/2376-127X.1000251

Page 5 of 5

27. Draper H (1996) Women, forced caesareans and antenatal responsibilities. J Med Ethics 22: 327-333.

28. ACOG (2005) Committee Opinion number 321.

29. (2013) AMA Position Statement on Maternal Decision making.

30. American Academy of Pediatrics Committee on Bioethics (1989) Fetal therapy: ethical considerations. Pediatrics 81: 898-899.
31. Royal College of Obstetricians and Gynaecologists (RCOG) Ethics Committee guidance (1993).

32. (2015) WHO. 